\title{
Relación morfométrica entre el tamaño del cuerpo y la longitud de huesos para estimar tamaño corporal en róbalo Eleginops maclovinus
}

Morphometric relationship between body size and bone length to estimate body size in Patagonian blenny Eleginops maclovinus

\section{Benjamín L. Suárez¹ y Ciro Oyarzún ${ }^{1}$}

\begin{abstract}
${ }^{1}$ Departamento de Oceanografía, Sección Pesquerías, Cabina 10 s/n, Barrio Universitario, Universidad de Concepción, Chile. bsuarez@udec.cl, coyarzun@udec.cl
\end{abstract}

\begin{abstract}
The knowledge and body size estimation of a prey item through undigested remains is important for the determination of prey's sizes selectivity by predator and understand these (predator-prey) interactions. Therefore, large size bones are used to perform these estimates, in part due to their resistance to digestion. The aim of this study is to determine the existence of bones with high correlation between them and body size in Eleginops maclovinus in order to estimate those parameters. Most of the measurements showed a highly significant correlation, but we decided only to work with the following bones: quadrate, suboperculum, cleithrum and maxilla because they showed the highest correlation coefficient. The length-weight relationship was: Weight $=0.041 \mathrm{Lt}^{2.597}$. We proposed that the selected measurements are suitable for morphometric analysis, and will help in a better understanding the predator-prey interactions, and potentially constitutes a good tool for studies of trophic relationship.
\end{abstract}

Key words: Biometry, osteology, trophic ecology, feeding, growth

Resumen.- El conocimiento y estimación del tamaño de un ítem presa a través de restos no digeridos es importante para la determinación de selectividad de tamaños por depredador y comprender dicha interacción (depredador-presa); es por esto que son utilizados huesos de gran dimensión para realizar dichas estimaciones, en parte por su resistencia a la digestión. El objetivo del presente trabajo fue determinar la existencia de huesos que presenten una alta correlación entre ellos y el tamaño del cuerpo en Eleginops maclovinus para estimar dichos parámetros. La mayoría de las mediciones presentaron una correlación altamente significativa, pero se decidió solo trabajar con los huesos cuadrado, subopércular, cleitro y maxilar ya que presentaron el mayor coeficiente de determinación. La relación longitud-peso fue: Peso = 0,041 $\mathrm{Lt}^{2,597}$. Se propone que las medidas seleccionadas son adecuadas para análisis morfométricos, pudiendo ayudar a entender mejor las interacciones depredador/presa y potencialmente constituye una herramienta útil para estudios de relaciones tróficas.

Palabras clave: Biometría, osteología, ecología trófica, alimentación, crecimiento

\section{INTRODUCCIÓN}

En estudios de ecología trófica, relación depredador-presa y evaluación de stocks explotados (Gosztonyi \& Kuba 1996), el conocimiento y estimación de la longitud y peso individual de los ítem presa a través de restos no digeridos en el contenido estomacal son de gran importancia para determinar la selectividad de tamaños del depredador y comprender su interacción con la presa (Newsome 1977, Hansel et al. 1988, Radke et al. 2000, Coop \& Kovác 2003, Gosztonyi et al. 2007).

Estructuras pequeñas, como los otolitos (Gosztonyi et al. 2007) presentan inconvenientes en la estimación del tamaño del ítem presa, al poder ser erosionados o disueltos totalmente (Tarkan et al. 2007) por los fluidos gástricos, lo que resulta en una identificación y medición incierta (Gosztonyi et al. 2007) provocando un sesgo en el tamaño de la presa (Jobling \& Breiby 1986, Tarkan et al. 2007). Estructuras duras con dimensiones mayores como ciertos huesos, presentan una mayor resistencia a la digestión (Hansel et al. 1988, Tarkan et al. 2007) y permanecen reconocibles por más tiempo (Mann \& Beaumont 1980).

Los huesos utilizados en este tipo de análisis tienen que presentar ciertas características, como ser especieespecíficos para la identificación de la especie, y presentar 
una correlación significativa para la estimación de longitud o peso. Los huesos craneales se caracterizan por ser útiles al momento de realizar la identificación del ítem presa (Coop \& Kovác 2003), además de presentar una buena relación biométrica (Tarkan et al. 2007). Es por esto, que los huesos más utilizados en este tipo de estudios son: dentario, maxilar, huesos faríngeos, opercular y parasfenoide; incluyendo también huesos de la cintura escapular y ciertas vértebras caudales (Newsome 1977, Gosztonyi \& Kuba 1996, Scharf et al. 1998, Radke et al. 2000, Coop \& Kovác 2003, Britton \& Shepherd 2005, Miranda \& Escala 2005, Gosztonyi et al. 2007, Tarkan et al. 2007).

El róbalo, Eleginops maclovinus Cuvier, 1830, es una especie que se distribuye desde Valparaíso, Chile $\left(33^{\circ} \mathrm{S}\right)$ y la región Patagónica de Argentina hasta Tierra del Fuego $\left(53^{\circ} \mathrm{S}\right)$ incluyendo a las Islas Malvinas (Gacitúa et al. 2008). Esta especie presenta un amplio rango de depredadores, desde aves marinas (Frere et al. 1996), mamíferos (Córdova et al. 2009, Sapoznikow et al. 2010) y algunos salmonídeos (Oyarzún ${ }^{1}$, datos no publicados), y que además, pertenece al grupo de especies recurso en Chile (SERNAPESCA 2011). En el presente trabajo fueron analizadas distintas medidas tanto de huesos craneales y de la cintura escapular, cintura pélvica (basipterigio) y endoesqueleto caudal con el objetivo de verificar la existencia de una relación significativa entre estos huesos y la longitud respectiva, ya sea estándar o total, con el fin de entregar información necesaria para estudios que se basen en relaciones biométricas.

\section{MATERIALES Y MÉTODOS}

Se muestreó un total de 21 ejemplares de E. maclovinus (entre 30 y $36 \mathrm{~cm}$ de longitud total, Lt), los cuales se obtuvieron a través de pesca artesanal en la desembocadura del río Bio-Bio $\left(36^{\circ} \mathrm{S}, 73^{\circ} \mathrm{O}\right)$. Estos se almacenaron a $-20^{\circ} \mathrm{C}$ para su posterior examen. En el laboratorio, con los individuos descongelados, se registró la longitud estándar (Ls), total (Lt) $( \pm 0,1 \mathrm{~cm})$, y peso total $( \pm 0,1 \mathrm{~g})$. Al momento de realizar las disecciones, los individuos se sometieron a inmersiones en agua hirviendo por pocos minutos (Radke et al. 2000, Copp \& Kovác 2003, Britton \& Shepherd 2005, Miranda \& Escala 2005, Tarkan et al. 2007) hasta que el tejido muscular fue extraído fácilmente al igual que los huesos a medir.

${ }^{1}$ Departamento de Oceanografía, Universidad de Concepción, Concepción, Chile. coyarzun@udec.cl
Se efectuaron mediciones (Fig. 1) $( \pm 0,01 \mathrm{~cm})$ en los huesos del lado izquierdo del ejemplar los que se mencionan a continuación: premaxilar, maxilar, palatino, dentario, ángulo-articular o angular, cuadrado, hiomandibular, opercular, subopercular, parasfenoide, frontal, cleitro (Gosztonyi \& Kuba 1996), basipterigio y endoesqueleto caudal.

\section{Análisis estadístico}

Se utilizó el programa SPSS Statistics 17.0 en el cual mediante un modelo de regresión lineal simple $(\mathrm{Lt}=\mathrm{a} \mathrm{x}+$ $\mathrm{b}$; donde $\mathrm{x}$ es la medida respectiva del hueso, a y b son los parámetros del modelo) se determinó la relación entre el tamaño del pez y las distintas mediciones realizadas para cada hueso con un intervalo de confianza del 95\%, destacando los que presentan mayor coeficiente de determinación $\left(\mathrm{R}^{2}\right)$.

El análisis se realizó a datos con y sin transformación logarítmica, ya que al ser transformados, presentarían una mejor adecuación a la linealidad del modelo aplicado (Copp \& Kovác 2003, Gosztonyi et al. 2007) permitiendo obtener un mayor ajuste. También se determinó la relación longitud-peso según la fórmula: Peso $=\mathrm{c} \mathrm{Lt}$; donde c y d son parámetros de la ecuación conocidos comúnmente como 'a' y 'b', los cuales en este trabajo son sustituidos con el fin de evitar confusión con los parámetros señalados anteriormente para el modelo lineal ya mencionado. Una vez realizada la regresión, se eligieron las medidas que presentaron un $\mathrm{R}^{2}$ más alto para a través de ellas estimar el peso total y comprobar la factibilidad del ensayo. Para obtener este resultado de manera directa, se fusionaron las 2 ecuaciones anteriores obteniéndose lo siguiente: Peso $_{\text {estimado }}=\mathrm{c}(\mathrm{a} \mathrm{X}+\mathrm{b})^{\mathrm{d}}$, donde $\mathrm{c} \mathrm{y} \mathrm{d} \mathrm{son}$ parámetros de la relación longitud-peso, a y b son los parámetros de la regresión lineal y X es la medida respectiva según el hueso utilizado. Posteriormente, se efectuó un ANOVA de un factor para determinar la existencia de diferencias significativas $(P<0,05)$ dentro del grupo (entre pesos promedios estimados y reales), y una vez comprobada la existencia de dichas diferencias, se realizó una prueba HSD de Tukey (Radke et al. 2000) para determinar qué grupo de pesos estimados fue distinto de los otros con el mismo nivel de significancia.

\section{RESUltados}

Los ejemplares muestreados $(n=21)$ presentaron una longitud media estándar y total de 29,0 $\pm 0,9$ y 33,8 $\pm 1,1$ $\mathrm{cm}$, respectivamente y un peso total promedio de 381,4 \pm 43,7 g (media \pm desviación estándar). El análisis de los datos con y sin transformación logarítmica presentó una 


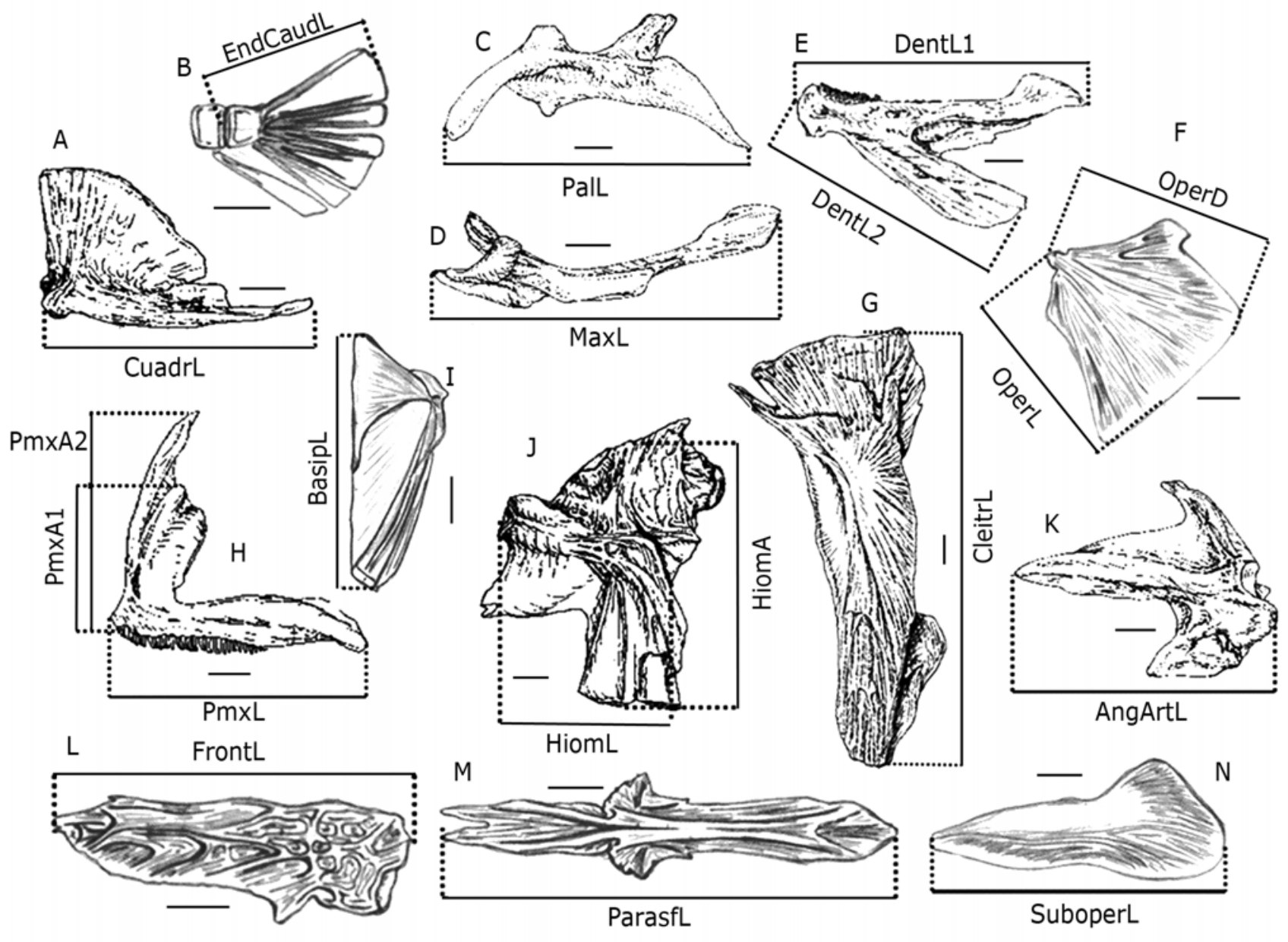

Figura 1. Esquema de las distintas mediciones realizadas en los huesos utilizados con sus respectivas abreviaturas para Eleginops maclovinus. A: Cuadrado, B: Endoesqueleto caudal, C: Palatino, D: Maxilar, E: Dentario, F: Opercular, G: Cleitro, H: Premaxilar, I: Basipterigio, J: Hiomandibular, K: Ángulo-Articular, L: Frontal, M: Parasfenoide y N: Subopercular. Barra = $5 \mathrm{~mm}$. Imágenes A-E, G-I modificadas de Falabella et al. (1995) / Schema of the different measurements on the bones used with their respective abbreviations for Eleginops maclovinus. A: Quadrate, B: Caudal Endoskeleton, C: Palatine, D: Maxilla, E: Dentary, F: Opercular, G: Cleithrum, H: Premaxilla, I: Basipterygium, J: Hyomandibular, K: Angle-articular, L: Frontal bone, M: Parasphenoid y N: Subopercular. Bar = $5 \mathrm{~mm}$. Images A-E, G-I modified from Falabella et al. (1995)

relación altamente significativa $(P \leq 0,001)$ para la mayoría de las mediciones y sus respectivas longitudes (Lt y Ls), a excepción de algunas medidas para el opercular (OperL) y el endoesqueleto caudal (EndCaudL) para datos con y sin transformación que fueron menos significativas ( $P \leq$ 0,05 ), pero igualmente válidas (Tabla 1 ). No obstante, algunas medidas sólo presentaron una relación altamente significativa para una sola longitud, como los frontales (FrontL para Ls en datos no transformados) y el hiomandibular (HiomA para Lt en datos con transformación). El palatino (PalL) fue el único en no presentar relación ya sea con o sin transformación logarítmica para ninguna longitud $(P \geq 0,05)$. Los coeficientes de determinación más elevados $\left(R^{2}>0,6\right)$ estuvieron ligados en mayor medida a la longitud total que a la longitud estándar. Sin embargo, las medidas que presentaron un $\mathrm{R}^{2}$ mayor que 0,6 tanto para Lt, como para Ls fueron Cuadrado (CuadrL), Subopérculo (SuboperL), Cleitro (CleitrL) y Maxilar (MaxL), por lo que estas medidas fueron las utilizadas para realizar la estimación del peso total. Sólo se trabajó con datos de longitud total debido a que presentaron $\mathrm{R}^{2}$ más altos que los de longitud estándar. 
Tabla 1. Regresiones lineales para cada medida de hueso utilizada en relación a la longitud (a y b son las constantes del modelo lineal con su respectivo $\mathrm{R}^{2}$ y significancia para cada longitud y transformación; las abreviaturas de los huesos están explicadas en la Fig. 1) / Linear regressions for each measure of bone utilized in relation to the length ( $a$ and $b$ are constants of the linear model with its respective $R^{2}$ and significance for each length and transformation, the abbreviations of the bones are explained in Fig. 1)

\begin{tabular}{|c|c|c|c|c|c|c|c|c|c|c|c|c|c|c|c|c|}
\hline \multirow[b]{3}{*}{ Variable } & \multicolumn{8}{|c|}{ Sin transformación } & \multicolumn{8}{|c|}{ Con transformación } \\
\hline & \multicolumn{4}{|c|}{ Longitud total $(\mathrm{cm})$} & \multicolumn{4}{|c|}{ Longitud estándar (cm) } & \multicolumn{4}{|c|}{ Longitud total $(\mathrm{cm})$} & \multicolumn{4}{|c|}{ Longitud estándar (cm) } \\
\hline & a & b & $\mathrm{R}^{2}$ & $P$ & a & b & $\mathrm{R}^{2}$ & $P$ & a & b & $\mathrm{R}^{2}$ & $P$ & a & $\mathrm{b}$ & $\mathrm{R}^{2}$ & $P$ \\
\hline PmxA1 & 19,58 & 16,67 & 0,58 & $\leq 0,001$ & 15,43 & 15,44 & 0,53 & $\leq 0,001$ & 0,51 & 1,56 & 0,61 & $\leq 0,001$ & 0,47 & 1,49 & 0,55 & $\leq 0,001$ \\
\hline PmxA2 & 10,23 & 20,48 & 0,49 & $\leq 0,001$ & 8,15 & 18,32 & 0,46 & $\leq 0,001$ & 0,40 & 1,48 & 0,51 & $\leq 0,001$ & 0,37 & 1,42 & 0,48 & $\leq 0,001$ \\
\hline PmxL & 11,85 & 17,08 & 0,56 & $\leq 0,001$ & 9,58 & 15,43 & 0,54 & $\leq 0,001$ & 0,51 & 1,45 & 0,58 & $\leq 0,001$ & 0,48 & 1,39 & 0,55 & $\leq 0,001$ \\
\hline MaxL & 12,36 & 10,30 & 0,76 & $\leq 0,001$ & 10,04 & 9,85 & 0,74 & $\leq 0,001$ & 0,70 & 1,33 & 0,77 & $\leq 0,001$ & 0,66 & 1,28 & 0,75 & $\leq 0,001$ \\
\hline DentL1 & 8,58 & 19,66 & 0,54 & $\leq 0,001$ & 6,71 & 17,88 & 0,48 & $\leq 0,001$ & 0,42 & 1,44 & 0,55 & $\leq 0,001$ & 0,39 & 1,38 & 0,49 & $\leq 0,001$ \\
\hline DentL2 & 8,99 & 18,61 & 0,53 & $\leq 0,001$ & 6,99 & 17,13 & 0,47 & $\leq 0,001$ & 0,45 & 1,43 & 0,54 & $\leq 0,001$ & 0,41 & 1,37 & 0,48 & $\leq 0,001$ \\
\hline Hiom A & 9,22 & 15,64 & 0,48 & $\leq 0,001$ & 7,09 & 14,96 & 0,42 & $\leq 0,001$ & 0,54 & 1,37 & 0,48 & $\leq 0,001$ & 0,49 & 1,32 & 0,42 & $\leq 0,050$ \\
\hline HiomL & 12,15 & 18,93 & 0,56 & $\leq 0,001$ & 9,62 & 17,16 & 0,52 & $\leq 0,001$ & 0,45 & 1,49 & 0,57 & $\leq 0,001$ & 0,42 & 1,43 & 0,52 & $\leq 0,001$ \\
\hline OperL & 5,59 & 21,79 & 0,33 & $\leq 0,050$ & 4,73 & 18,76 & 0,35 & $\leq 0,050$ & 0,36 & 1,41 & 0,35 & $\leq 0,050$ & 0,35 & 1,34 & 0,36 & $\leq 0,050$ \\
\hline OperD & 7,42 & 15,68 & 0,56 & $\leq 0,001$ & 5,84 & 14,69 & 0,51 & $\leq 0,001$ & 0,54 & 1,32 & 0,58 & $\leq 0,001$ & 0,49 & 1,27 & 0,52 & $\leq 0,001$ \\
\hline SuboperL & 6,42 & 13,82 & 0,69 & $\leq 0,001$ & 5,08 & 13,15 & 0,63 & $\leq 0,001$ & 0,59 & 1,24 & 0,68 & $\leq 0,001$ & 0,54 & 1,19 & 0,62 & $\leq 0,001$ \\
\hline PalL & 0,78 & 32,31 & 0,05 & $\geq 0,050$ & 0,94 & 27,12 & 0,10 & $\geq 0,050$ & 0,06 & 1,51 & 0,06 & $\geq 0,050$ & 0,08 & 1,44 & 0,12 & $\geq 0,050$ \\
\hline CuadrL & 9,71 & 19,73 & 0,65 & $\leq 0,001$ & 8,17 & 17,09 & 0,68 & $\leq 0,001$ & 0,41 & 1,46 & 0,65 & $\leq 0,001$ & 0,40 & 1,40 & 0,67 & $\leq 0,001$ \\
\hline AngArtL & 8,36 & 19,99 & 0,51 & $\leq 0,001$ & 6,81 & 17,69 & 0,50 & $\leq 0,001$ & 0,42 & 1,44 & 0,52 & $\leq 0,001$ & 0,40 & 1,38 & 0,51 & $\leq 0,001$ \\
\hline CleitrL & 5,12 & 10,50 & 0,69 & $\leq 0,001$ & 3,93 & 11,03 & 0,60 & $\leq 0,001$ & 0,69 & 1,07 & 0,70 & $\leq 0,001$ & 0,62 & 1,05 & 0,61 & $\leq 0,001$ \\
\hline ParasfL & 4,32 & 14,47 & 0,64 & $\leq 0,001$ & 3,35 & 13,96 & 0,57 & $\leq 0,001$ & 0,57 & 1,16 & 0,65 & $\leq 0,001$ & 0,52 & 1,13 & 0,58 & $\leq 0,001$ \\
\hline FrontL & 4,02 & 22,51 & 0,39 & $\leq 0,050$ & 3,45 & 19,24 & 0,42 & $\leq 0,001$ & 0,30 & 1,39 & 0,35 & $\leq 0,050$ & 0,31 & 1,32 & 0,40 & $\leq 0,050$ \\
\hline BasipL & 6,36 & 15,89 & 0,64 & $\leq 0,001$ & 4,72 & 15,64 & 0,52 & $\leq 0,001$ & 0,54 & 1,29 & 0,65 & $\leq 0,001$ & 0,47 & 1,25 & 0,53 & $\leq 0,001$ \\
\hline EndCaudL & 3,92 & 28,11 & 0,30 & $\leq 0,050$ & 3,11 & 24,42 & 0,28 & $\leq 0,050$ & 0,15 & 1,51 & 0,26 & $\leq 0,050$ & 0,14 & 1,44 & 0,25 & $\leq 0,050$ \\
\hline
\end{tabular}

La relación longitud-peso (Fig. 2) según la ecuación potencial obtenida fue: Peso $=0,041 \mathrm{Lt}^{2,597}\left(\mathrm{R}^{2}=0,577 ; P \leq\right.$ 0,001). No obstante, Licandeo et al. (2006) obtuvieron valores distintos para las constantes (Peso $=0,0098 \mathrm{Lt}^{3}, 03$, $\left.\mathrm{R}^{2}=0,99, \mathrm{n}=205\right)$. Es por esto, que se decidió estimar el peso a través de ambas fórmulas de longitud-peso (Tabla 2) con sus respectivos parámetros, pero manteniendo las constantes obtenidas en el presente trabajo para la relación lineal entre las medidas hueso-longitud (Tabla 1).

Independientemente de la ecuación longitud-peso utilizada, el peso promedio estimado a partir de cualquiera de las medidas de los huesos propuestos es casi idéntico, lo que refleja una relación significativa entre la medida del hueso y la longitud (Tabla 2). El ANOVA realizado demuestra la existencia de diferencias entre los grupos $(F$ $=33,963, P<0,05$ ) (pesos promedios reales, pesos estimados a partir de los huesos y pesos obtenidos con la ecuación de Licandeo et al. 2006), mientras que la prueba HSD de Tukey establece que no hubo diferencias significativas $(P>0,05)$ entre el peso promedio real, y los

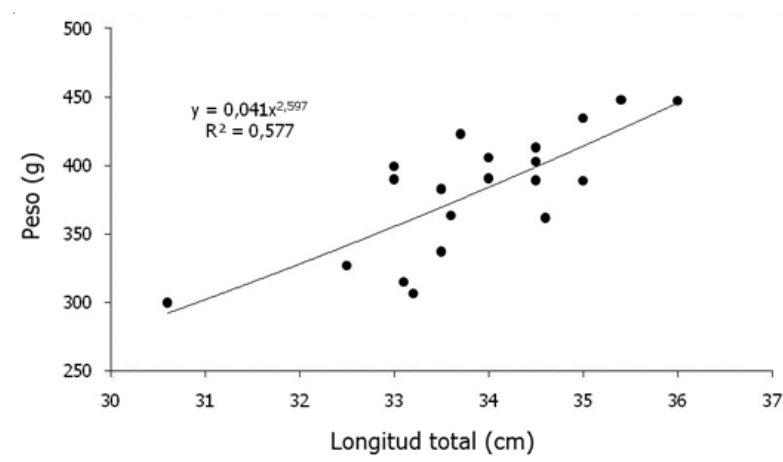

Figura 2. Relación longitud-peso para los ejemplares muestreados de Eleginops maclovinus $(n=21)$ con su respectiva ecuación y coeficiente de determinación / Length-weight relationship for specimens sampled $(n=21)$ with respective equation and determination coefficient

pesos promedios obtenidos a partir de la relación longitud-peso propuesta en el trabajo actual. Esto difiere de los pesos promedios obtenidos según la ecuación de Licandeo et al. (2006) $(P<0,05)$. 


\section{Discusión}

En otros estudios que comparan la longitud de los huesos con la longitud de los organismos es común observar valores de coeficiente de determinación entre 0,8 y 0,9 (Copp \& Kovác 2003, Gosztonyi et al. 2007). No obstante, en el presente estudio los valores más altos estuvieron entre 0,60 y 0,77 . Otros autores como Britton \& Shepherd (2005) y Miranda \& Escala (2005) trabajaron con tamaños de muestra mayores a los examinados en este trabajo $(n=$ 21), lo que podría explicar la menor relación. Sin embargo, otros reportes con tamaños menores de muestra dan información igualmente válida (Plug 2008). El acotado rango de longitudes utilizado (Lt: 30,6-36 cm, Ls: 26,5$30,5 \mathrm{~cm}$ ) también puede explicar en parte lo encontrado, no obstante, la relación fue altamente significativa ( $P \leq$ 0,001).

En el presente trabajo, de todos los huesos analizados sólo se seleccionaron 4 (Tabla 2) para llevar a cabo la estimación del tamaño del cuerpo debido a su relación altamente significativa y por presentar el mayor coeficiente de determinación. En otros trabajos, se destaca la utilidad de huesos distintos a los presentados en este estudio, ya sea para el reconocimiento de especies, como para la estimación del tamaño de la presa ingerida (Newsome 1977, Radke et al. 2000, Tarkan et al. 2007), lo que naturalmente es propio de la especificidad de cada taxón, pudiendo diferir entre especies o incluso dentro del mismo género (Plug 2008). Estructuras de menor tamaño, como los otolitos presentan inconvenientes por una mayor erosión (Jobling \& Breiby 1986) por lo que es aconsejable trabajar con estructuras

Tabla 2. Peso promedio estimado a través de las distintas ecuaciones longitud-peso descritas, con sus respectivos parámetros, manteniendo las constantes de relación lineal propuestas para cada medida de hueso (Porcentaje de variación: \%Var $=\left[\left(P_{\text {est }}-P_{\text {real }}\right) / P_{\text {real }}\right.$ ] * 100 / Average weight estimated through the different lengthweight equations described above, with their respective parameters, keeping the constant of the linear relationship as proposed for each bone (Percentage of variation: \%Var $=\left[\left(\mathrm{P}_{\text {est }}{ }^{-}\right.\right.$ $\left.\left.P_{\text {real }}\right) / P_{\text {real }}\right] * 100$

Trabajo Actual Licandeo et al. (2006)

\begin{tabular}{lrr}
\hline Peso Real & 381,39 & \\
CuadrL & 385,01 & 423,14 \\
SuboperL & 385,03 & 423,17 \\
CleitrL & 385,05 & 423,21 \\
MaxL & 385,08 & 423,27 \\
\% Var. Prom. & 0,95 & 9,88 \\
\hline
\end{tabular}

de dimensiones mayores al momento de realizar la estimación del tamaño del cuerpo. Los huesos seleccionados en este trabajo presentan un tamaño adecuado para el estudio, ya que si bien el cuadrado y maxilar son los más pequeños dentro de la selección, siguen presentando dimensiones mayores a las observables en un otolito u otras estructuras (Tarkan et al. 2007). Las diferencias con la relación longitud-peso descrita por Licandeo et al. (2006) se explicaría por el rango de longitudes considerado en cada uno de los estudios (Licandeo et al. 2006: 16-58 cm Lt; Trabajo actual: 30-36 cm Lt). No obstante, según los pesos promedios estimados bajo ambas ecuaciones (longitud-peso), se comprueba una alta relación obtenida para las medidas de los huesos propuestos, ya que todos los pesos promedios estimados se encuentran con valores muy cercanos entre sí (según la ecuación utilizada; variación $<1 \%$ ). La diferencia de promedios para los pesos estimados en la Tabla 2 según el modelo utilizado, se atribuye netamente a la diferencia de los parámetros de dicho modelo (longitud-peso).

La estimación del tamaño del cuerpo no necesariamente se puede obtener desde restos estomacales, también existen trabajos en los cuales es posible calcular dicha estimación a partir de restos arqueológicos proporcionando puntos de vista tanto en los patrones de depredación, como en la explotación estacional y las condiciones ambientales (Plug 2008).

Según lo obtenido, se acepta y propone que las medidas de los huesos seleccionados (CuadrL, SuboperL, CleitrL y MaxL) son adecuadas para análisis morfométricos, y potencialmente constituyen una herramienta útil para estudios de las relaciones tróficas que incluyan restos de peces como parte de la dieta.

\section{Agradecimientos}

Los autores desean expresar su gratitud hacia los tres revisores anónimos por las sugerencias y comentarios aportados para la mejora del presente manuscrito.

\section{LITERATURA CITADA}

Britton J \& J Shepherd. 2005. Biometric data to facilitate the diet reconstruction of piscivorous fauna. Folia Zoology 54(1-2): 193-200.

Coop G \& V Kovác. 2003. Biometric relationships between body size and bone lengths in fish prey of the Eurasian otter Lutra lutra: chub Leuciscus cephalus and perch Perca fluviatilis. Folia Zoology 52(1): 109-112. 
Córdova O, J Rau, C Suazo \& A Arriagada. 2009. Estudio comparativo de la ecología alimentaria del depredador de alto nivel trófico Lontra felina (Molina, 1782) (Carnivora: Mustelidae) en Chile. Revista de Biología Marina y Oceanografía 44(2): 429-438.

Falabella F, R Meléndez \& M Vargas. 1995. Claves osteológicas para peces de Chile central. Un enfoque arqueológico, 208 pp. Artegrama, Santiago.

Frere E, E Gandini \& V Lichtschein. 1996. Variación latitudinal en la dieta del pingüino de Magallanes (Spheniscus magellanicus) en la costa patagónica argentina. Ornitología Neotropical 7: 35-41.

Gacitúa S, C Oyarzún \& R Veas. 2008. Análisis multivariado de la morfometría y merística del robalo Eleginops maclovinus (Cuvier, 1830). Revista de Biología Marina y Oceanografía 43(3): 491-500.

Gosztonyi A \& L Kuba. 1996. Atlas de huesos craneales y de cintura escapular de peces costeros patagónicos. Fundación Patagonia Natural Puerto Madryn, Informe Técnico 4: 1-32.

Gosztonyi A, L Kuba \& L Mansur. 2007. Estimation of body size using morphometric relationships of head bones, pectoral fin bones and bony precaudal distance in Raneya brasiliensis (Kraup, 1856) (Pisces, Ophidiiformes, Ophidiidae) in Patagonian waters. Revista de Biología Marina y Oceanografía 42(1): 1-5.

Hansel H, S Duke, P Lofy \& G Gray. 1988. Use of diagnostic bones to identify and estimate original lengths of ingested prey fishes. Transactions of the American Fishery Society 117: 55-62.

Jobling M \& A Breiby. 1986. The use and abuse of fish otoliths in studies of feeding habits of marine piscivores. Sarsia 71: 265-274.

Licandeo R, C Barrientos \& M González. 2006. Age, growth rates, sex change and feeding habits of notothenioid fish Eleginops maclovinus from the central-southern Chilean coast. Environmental Biology of Fishes 77: 51-61.
Mann R \& W Beaumont. 1980. The collection, identification and reconstruction of lengths of fish prey from their remains in pike stomachs. Fisheries Management 11: 169-172.

Miranda R \& M Escala. 2005. Morphometrical comparison of cleithra, opercular and pharyngeal bones of autochthonous Leuciscinae (Cyprinidae) of Spain. Folia Zoology 54(1-2): 173-188.

Newsome G. 1977. Use of opercular bones to identify and estimate lengths of prey consumed by piscivores. Canadian Journal of Zoology 55: 733-736.

Plug I. 2008. Fish bone sizes as estimators of standard lengths of three southern African freshwater species with application to archaeological samples: a preliminary investigation. South African Journal of Science 104: 71-75.

Radke R, T Petzoldt \& C Wolter. 2000. Suitability of pharyngeal bone measures commonly used for reconstruction of prey fish length. Journal of Fish Biology 57: 961-967.

Sapoznikow A, C Reeves, G Sessa, L Mansur \& M de la Reta. 2010. Mamíferos marinos. Fundación Patagonia natural. 46 pp. [en línea] <http://www.patagonianatural.org/ attachments/190_Mamiferos\%20Marinos.pdf>

SERNAPESCA. 2011. Anuarios estadístico de pesca. Servicio Nacional de Pesca, Valparaíso. <http://www.sernapesca.cl/ index.php?option=com_remository\&Itemid=54\&func=select\&id=2>

Scharf F, R Yetter, A Summers \& F Juanes. 1998. Enhancing diet analyses of piscivorous fishes in the Northwest Atlantic through identification and reconstruction of original prey sizes from ingested remains. Fishery Bulletin 96(3): 575588.

Tarkan A, C Gürsoy-Gaygusuz, O Gaygusuz \& H Acipinar. 2007. Use of bone and otolith measures for size-estimation of fish in predator-prey studies. Folia Zoology 56(3): 328-336.

Recibido el 13 de junio de 2012 y aceptado el 18 de noviembre de 2012

Editor: Claudia Bustos D. 